\title{
Schwellenkonzepte als Zugänge fachlichen Verstehens - Wege zur differenzierten Erschließung komplexer (ökonomischer) Basiskonzepte im GW-Unterricht
}

\author{
*felix.magnus.bergmeister@univie.ac.at, Institut für Geographie \& Regionalforschung, Universität Wien
}

eingereicht am: 13.06.2017, akzeptiert am: 25.07.2017

Dieser Artikel führt in den lerntheoretischen Ansatz der Schwellenkonzepttheorie ein und umreißt, wie dieses konstruktivistische Lernkonzept zur Erschließung der neuen Basiskonzepte im österreichischen GW-Unterricht genutzt werden kann. Schwellenkonzepte stellen dabei gedankliche Türöffner dar, die Zugänge in komplexe disziplinspezifische Denkweisen eröffnen und somit Lernenden ein umfangreicheres Verständnis der Basiskonzepte ermöglichen können. Der Beitrag skizziert abschließend eine Unterrichtssequenz, die für die Erarbeitung von Schwellenkonzepten geeignet scheint.

Keywords: Schwellenkonzepte, Basiskonzepte, GW-Unterricht, Ökonomie, Geographie, Kontingenz, Unterrichtspraxis

\section{Stepping stones to basic concepts - Threshold concepts as gateways to comprehensive disciplinary thinking in the Austrian geography and economic education classroom}

This article introduces the learning theory of Threshold Concepts and explores how they might help learners gain a broader and more comprehensive understanding of the Basic Concepts outlined in the new Austrian curriculum. Threshold concepts are conceived as breakthrough concepts and can function as door openers to more complex discipline-specific ways of thinking. A teaching activity is outlined to illustrate how this form of pedagogy may be turned into practice.

Keywords: threshold concepts, basic concepts, geography and economic education, contingency, lesson planning, teaching, conceptual change

\section{$1 \quad$ Einleitung}

„Beim iPhone sieht man, wie eine globale Wertschöpfungskette funktioniert - vom Designentwurf und Marketing, über Zulieferung und Produktion bis zu Vertrieb und Recycling. Man erkennt auch wie unsolidarisch das alles aufgebaut ist ... weil die Arbeiter ja fast nichts verdienen, obwohl das Handy extrem teuer ist."

Dieser Kommentar wurde kürzlich von einem zweitsemestrigen Studierenden im Proseminar ,Einführung in den kompetenzorientierten GW-Unterricht' am Institut für Geographie und Regionalforschung der Universität Wien geäußert. Eben wurden Klafkis (1994) Schlüsselprobleme besprochen und die Studierenden überlegten, wie ein lebensweltorien- tierter exemplarischer Anknüpfungspunkt zu globalen Herausforderungen wie Verteilungsgerechtigkeit und/oder Technikfolgen im GW-Unterricht aussehen könnte. Es war naheliegend, an ein elektronisches Gerät aus globaler Produktion zu denken - was an der Wortmeldung jedoch bemerkenswert erscheint, ist der Gedankenschritt der doppelten Abstraktion, den sie beschreibt. Der Sprecher nimmt ein beliebtes Konsumgut zum Ausgangspunkt und verortet dieses ursächlich anhand des wirtschaftsgeographischen Konzepts der globalen Wertschöpfungskette. Durch diese theoretische Abstraktion eröffnet sich eine fachspezifische Sichtweise, die den Studierenden dabei unterstützt, Phänomene und Problemstellungen aus geographisch-ökonomischer Perspektive kausal zu begreifen. Dieser Bereich der gedanklichen Erschließung lässt sich dem technischen und praktischen Erkenntnis- und Vermittlungsinteresse zuordnen, durch 
das,Wenn-Dann'-Rationalitäten anhand verfügbarer fachwissenschaftlicher Erklärungsansätze sichtbar gemacht werden (vgl. Vielhaber 1999). Der junge Kollege in unserem Beispiel bleibt jedoch nicht auf der faktischen Erklärungsebene, sondern beurteilt die potentiellen sozialen Auswirkungen, die sich durch weltwirtschaftliche Arbeitsteilung, den Wettbewerb der internationalen Zulieferer und die Ausweitung des globalen Niedriglohnsektors ergeben kritisch. Diese zweite Abstraktionsebene verlässt technisch-zweckrationale Erklärungspfade und stellt das solidarische Bildungs- und Gesellschaftsinteresse an Mündigkeit und kritisch-emanzipatorischer Selbstbestimmung in den Vordergrund (vgl. Hedtke 2015; Vielhaber 1988). Fragen um Interessen, Konflikte und Macht erscheinen vor dem Hintergrund von Diversität und Disparität. Das ermöglicht die Reflexion von Handlungssituationen und Verwertungszusammenhängen auf persönlicher, regionaler und globaler Ebene.

\section{Vom Desiderat der multiperspektivischen Erschließung komplexer Wirklichkeiten}

Die hier beispielhaft umrissene doppelte Abstraktion im Zuge der kognitiven Erschließung von Wirklichkeit ist ein wesentliches Desiderat zukunftsfähiger wirtschaftlicher Allgemeinbildung im Fach GW. Es geht darum zu erkennen, dass die Probleme des 21. Jahrhunderts durch eine große faktische und ethische Komplexität gekennzeichnet sind und daher fachliche und moralische Fragen nebeneinandergestellt werden müssen (vgl. Mehren et al. 2015). Lernende sollen dazu befähigt werden, die Limitationen und Interessensorientierungen fachwissenschaftlicher Erklärungsansätze zu enttarnen und zudem zur grundlegenden Einsicht gelangen, dass die vermeintlich, wirkliche' Wirklichkeit auch eine ganz andere sein kann (vgl. Rhode-Jüchtern 2011). Zentral ist hierbei die Akzeptanz von Multiperspektivität und Kontingenz. Die Ökonomie ist keine unfehlbare und wertneutrale Wissenschaft, die durch ihre ,bewährten Methoden, Modelle und Heuristiken zu dauerhaftem Wirtschaftswachstum und sozialer Gerechtigkeit beiträgt (vgl. Bischoff 2009: 66f). Wirtschaftliche Phänomene und die ihnen zugrundeliegenden institutionellen und politischen Rahmenbedingungen sind durch hochgradige Komplexität gekennzeichnet und daher nicht ohne die Wechselwirkung aus sozialen, historischen und politischen Entwicklungen zu erklären (vgl. Piketty 2015). Zeitgemäße ökonomische Bildung verlangt dementsprechend nach einem perspektivischen Pluralismus, der sich nicht auf neoklassische Marktund Entscheidungsmodelle beschränkt, sondern sich an lebensweltbezogenen sozioökonomischen Heraus- forderungen orientiert (vgl. Weber 2015). Im allgemeinbildenden österreichischen Schulsystem kommt dem GW-Unterricht diesbezüglich eine zentrale Rolle zu. Neben der Vermittlung von ökonomischen und geographischen Fachinhalten soll er Schüler/innen zu mehr Autonomie und Selbstbestimmung verhelfen:

"Es gilt die Schülerlinnen in einer Zeit des globalen Wandels entscheidungs- und handlungsfähig für die Zukunft zu machen. [...] Dies beinhaltet den kompetenten Umgang mit wesentlichen komplexen Fachinhalten und Fachmethoden, die sich an den bei den Schülerlinnen ausgeprägten Motivationen, Interessen und Bedürfnissen orientieren und dem letzten Stand der fachlichen und fachdidaktischen Erkenntnisse entsprechen. [...] [Dabei die] Existenz verschiedener interessensgeleiteter Wirklichkeiten von der lokalen bis zur globalen Ebene aufzuzeigen, zu vergleichen, zu bewerten und kritisch $z u$ hinterfragen, ist Ziel der Multiperspektivität" (Hinsch et al. 2014: 51f.)

Wie dieses Zitat verdeutlicht, geht es stets um die dialektische Erschließung von polyphonen Wirklichkeiten entlang einer orthodoxen und einer heterodoxen Dimension. Einerseits sollen die diskursiven und materiellen Konstruktionsmechanismen der bestehenden Welt(un)ordnungen begriffen werden und andererseits sollen sie als kontingent identifiziert und kritisch hinterfragt werden. Wie Fridrich \& Hofmann-Schneller (2017: 55) diesbezüglich betonen, bedarf es der Grundlage einer breiten sozioökonomischen Wissensbasis, um sich in hochkomplexen, wirtschaftlich geprägten Zusammenhängen zu orientieren. Programmatisches und berufsspezifisches Wirtschaftsund Finanzwissen alleine reicht dafür jedoch nicht aus. Was notwendig scheint, ist eine subjektsensible sozioökonomische Bildung, die ein tiefgründigeres Verstehen von wirtschaftlichen Zusammenhängen befördert, jungen Menschen zu einer ethisch fundierten Bewertung ihrer Umwelt verhilft und sie zum Denken in Alternativen sowie zu verantwortungsbewusstem Handeln ermutigt (vgl. Hedtke 2015).

Der 2016 verordnete Lehrplan für die Sekundarstufe II der AHS (BGBl. 217/2016) unterstützt diese Ansprüche durch konsequenten Lebensweltbezug und die Betonung von Kontingenz. Fachliche Basiskonzepte unterstützen dabei die Handlungs- und Kompetenzorientierung und stellen sicher, dass anwendungsbezogenes Wissen gefördert wird:

„Basiskonzepte bündeln fachliche Zugänge, die darauf abzielen, eine unübersichtliche komplexe Welt für Schülerinnen und Schüler lesbar und verhandelbar zu machen. Durch die Brille der Basiskonzepte betrachtet, lernen Schüler/innen Sachverhalte in geographischer und ökonomischer Perspektive zu (de)konstruieren, zu strukturieren, zu problematisieren und hieraus reflektierte Handlungen zu entwickeln" (BGBl. 217/2016: 60f.). 
Durch Basiskonzepte soll der GW-Unterricht außerdem stärker an den aktuellen Stand der zugrundeliegenden Basiswissenschaften angebunden werden. Es geht darum, den fachlichen Kern der ökonomischen und geographischen Bildung zu konkretisieren und die Lernziele des Lehrplans aus inhaltlicher und methodischer Sicht zu ergänzen (vgl. Hinsch et al. 2014: 52). Aufgrund ständiger gesellschaftlicher, fachwissenschaftlicher und fachdidaktischer Weiterentwicklung ist die Liste der Basiskonzepte notwendigerweise unvollständig und bedarf kontinuierlicher Anpassung und Erweiterung. Die folgenden $13 \mathrm{Ba}-$ siskonzepte werden für den GW-Unterricht aktuell aus dem fachwissenschaftlichen und fachdidaktischen Diskurs abgeleitet (BGBl. 217/2016):

1. Raumkonstruktion und Raumkonzepte

2. Regionalisierungen und Zonierungen

3. Diversität und Disparität

4. Maßstäblichkeit

5. Wahrnehmung und Darstellung

6. Nachhaltigkeit und Lebensqualität

7. Interessen, Konflikte, Macht

8. Arbeit, Produktion und Konsum

9. Märkte, Regulierung und Deregulierung

10. Wachstum und Krise

11. Mensch-Umwelt-Beziehungen

12. Geoökosystem

13. Kontingenz

Es wird deutlich, dass die Basiskonzepte generell großen inhaltlichen Spielraum zulassen und auf die grobe' fachwissenschaftliche Erschließungsrichtung hinweisen. Das Basiskonzept Märkte, Regulierung und Deregulierung dient beispielsweise als disziplinspezifische Orientierung, um das potentielle Spannungsfeld zwischen Märkten und Institutionen auf Grundlage wirtschafts- und sozialwissenschaftlicher Erkenntnisse im Unterricht zu befördern. Die Schüler/innen sollen komplexe Sachverhalte durch die Kenntnis und Anwendung geeigneter Grundkonzepte, Theorien und Modelle begreifen und dabei lernen, wie Ökonominnen und Ökonomen (zu) denken. Sie sollen aber gleichzeitig auch zur Kritik an der Fachwissenschaft befähigt werden. Im hier genannten Beispiel wäre es dementsprechend sinnvoll, Kenntnisse aus der Marktformenlehre und Preistheorie vor dem Hintergrund wirtschaftspolitischer Interessenslagen zu reflektieren und dadurch institutionelle und individuelle Entscheidungen wissenschaftlich fundiert erklärbar und kritisierbar zu machen. Die Basiskonzepte des semestrierten Lehrplans bieten dazu einen geeigneten Rahmen, für die Unterrichtspraxis GW scheint es jedoch sinnvoll, die Erschließbarkeit der einzelnen Basiskonzepte genauer zu untersuchen. Der Zugriff auf sogenannte ,Threshold Concepts' oder Schwellenkonzepte ist aus lerntheoretischer Sicht vielversprechend.

\section{Schwellenkonzepte als Türöffner des Verstehens}

Schwellenkonzepte beschreiben einen innovativen lerntheoretischen Ansatz, der auf Meyer \& Land (2003; 2005) zurückgeht. Die Autoren gehen davon aus, dass es in allen wissenschaftlichen Disziplinen kontraintuitive Hürden oder eben Schwellen des Verstehens gibt, die - sobald sie überwunden werden fachspezifisches Denken zulassen und damit völlig neue Sichtweisen auf die Welt ermöglichen:

"A threshold concept can be considered as akin to a portal, opening up a new and previously inaccessible way of thinking about something. It represents a transformed way of understanding, or interpreting, or viewing something without which the learner cannot progress. As a consequence of comprehending a threshold concept there may thus be a transformed internal view of subject matter, subject landscape, or even world view. This transformation may be sudden or it may be protracted over a considerable period of time, with the transition to understanding proving troublesome. Such a transformed view or landscape may represent how people 'think' in a particular discipline, or how they perceive, apprehend, or experience particular phenomena within that discipline (or more generally)" (Meyer \& Land 2003: 1).

Schwellenkonzepte sind also kognitive Arrangements, die es Individuen ermöglichen, über bestimmte Sachverhalte, Probleme und Phänomene in fachlich-differenzierter Art und Weise nachzudenken, bzw. die daraus gewonnenen Einsichten als Grundlage für weitere problemorientierte Erkenntnis- und Handlungsprozesse exemplarisch heranzuziehen. Dabei helfen Schwellenkonzepte, die Ebene des rein deklarativen Wissens zu überwinden und Lernenden zu prozedural einsetzbaren Kenntnissen und Fertigkeiten zu verhelfen (Davies \& Mangan 2007: 711). Wie Perkins (2008: 4) beschreibt, fördern Schwellenkonzepte die Aneignung von handlungsorientierten und proaktiven (kreativen) Wissensformen. Individuen werden dadurch einerseits befähigt, erworbenes Wissen kontextspezifisch und situationsadäquat anzuwenden und es andererseits bei Bedarf als Ausgangspunkt für die Generation neuen Wissens heranzuziehen. Schwellenkonzepte können daher sowohl als ,Station' als auch als ,Prozess' verstanden werden. Einerseits beschreiben sie einen kognitiven Entwicklungsschritt, den ein Individuum geleistet hat und andererseits fungieren sie als Ausgangsbasis für neue Verknüpfungen und Wege des Verstehens (Walker 2013: 248). Weiters sind im Rahmen der Schwellenkonzepttheorie verstandene Lernprozesse mit tiefgreifenden ontologischen und epistemologischen Veränderungen verbunden. Wie Cousin (2008: 264) aufzeigt, bewegen sich Lernende im Spannungsfeld der Dialektik aus Wissen und 
Sein. Die Erfahrung von Schwellenkonzepten verändert sowohl was sie über die Welt wissen, als auch die Art und Weise, wie sie sich vor diesem Hintergrund selbst erkennen und verorten. Meyer \& Land (2003) nennen drei Beispiele für Schwellenkonzepte aus unterschiedlichen Disziplinen: Opportunitätskosten aus der Ökonomie, Signifikation aus den Cultural Studies und Limit aus der Mathematik. Ein typisches Beispiel aus dem Bereich von GW ist das einleitend vorgestellte Konzept der (globalen) Wertschöpfungskette (vgl. Fouberg 2013). Ohne das Verständnis dieses Schwellenkonzepts ist es schwer vorstellbar, das Phänomen der Globalisierung aus der Sicht von Geographinnen und Geographen zu begreifen und entsprechenden Zugang $\mathrm{zu}$ raumwissenschaftlich und ökonomisch begründbaren Denk- und Kritikweisen zu erlangen.

Schwellenkonzepte weisen nach (Meyer \& Land 2003) zumeist fünf wichtige Merkmale auf, die sie für den Unterricht in Geographie und Wirtschaftskunde besonders bedeutsam machen. Wie Davies \& Mangan (2007: 712) anmerken, sind diese Merkmale unterschiedlich stark miteinander verbunden und nicht bei jedem Schwellenkonzept im selben Maße ausgeprägt.

1. Sie sind transformativ, wenn sie einmal verstanden wurden, tragen sie auch zu einer veränderten Wahrnehmung der Welt bei. So würde es die Einsicht in den globalen Wertschöpfungskomplex eines Handys voraussichtlich erschweren, Elektronikgeräte ausschließlich aus der (naiven) individuellen Konsumperspektive zu beurteilen.

2. Sie sind potentiell irreversibel. Es scheint schwer vorstellbar, dass durch Schwellenkonzepte erweiterte individuelle Perspektiven zu einem späteren Zeitpunkt wieder auf alte, selbst als unzureichend erkannte Denkmuster eingeschränkt werden.

3. Sie sind integrativ und lassen auf diese Weise die Komplexität von möglichen Beziehungsgeflechten erkennen, die im gewohnten Alltagsdenken mitunter verborgen bleiben.

4. Sie sind grenzorientierend. Beispielsweise stellt das Konzept der Wertschöpfungskette ein wichtiges ökonomisches Konzept dar, seine notwendige Vernetzung mit sozialen, politischen und kulturellen Fragestellungen (der Lebensweltbezug) stellt aber auch gleichzeitig die Grenzen disziplinären Denkens in Frage beziehungsweise definiert sie neu.

5. Sie sind beschwerlich, da sie oft kontraintuitiv sind und vermeintlich richtige und einfache Weltbilder in ihrer verkürzten Singularität erschüttern und dazu anspornen, die Welt auf andere und komplexere Weise zu verstehen.

Schwellenkonzepte sind aufgrund ihrer transformativen Charakteristik der Conceptual Change Theorie des Lernens zuzuordnen (vgl. Carey 1991). Der Konzept- wechsel Ansatz beschreibt, dass Lernende bestehendes Wissen und Alltagserfahrungen bei Bedarf grundlegend revidieren müssen, um neue Strukturen aufzubauen, bzw. um neue Aspekte zu integrieren (Jonen, Möller \& Hardy 2003: 95). Laut Davies \& Mangan (2007: 715) können im Bereich der ökonomischen Schwellenkonzepttheorie drei wesentliche Konzeptwechsel unterschieden werden. Erstens, die Integration von ökonomischem Grundverständnis in die Denkund Alltagswelten der Lernenden (z. B. der Unterschied zwischen Preis und Kosten). Zweitens, eine tiefgreifende Vernetzung dieser Grundideen zu fachspezifischen Erklärungsansätzen (z. B. Opportunitätskosten) und drittens, eine höherstufige Synthese disziplinärer Denkmuster zu Modellen und Theorien (z. B. Preiselastizität). Es ist wichtig, dass erst die Stufen zwei und drei als ,echte' disziplinäre Schwellenkonzepte zu verstehen sind, da sie durch ihren höheren integrativen Anspruch die eigentlichen Türöffner zu vernetztem, fachspezifischen Denken sind (vgl. ebd.). Die Stufe eins hingegen stellt eine fundamentale Schnittstelle zwischen Vorwissen und Fachwissen dar und ist daher für den GW-Unterricht ebenfalls besonders relevant. Um beispielsweise das Schwellenkonzept der Opportunitätskosten zu erschließen muss erst einmal geklärt werden, was Preis und Kosten aus ökonomischer Sicht überhaupt bedeuten, beziehungsweise ob und wie sich diese Bedeutung vom Vorwissen der Lernenden unterscheidet. Dazu sollte im Sinne der Kontingenz ebenfalls ein kritischer Impuls gesetzt werden, der mögliche Grenzen dieser Begrifflichkeiten aufzeigt. Diese Überlegungen eröffnen die Frage, welche Schwellenkonzepte ${ }^{1}$ für den GW-Unterricht besonders relevant sind und wie diese aus praktischer Sicht im Unterricht erfahrbar gemacht werden können. Gute Orientierungshilfen hierfür sind, wie bereits angedeutet, die Basiskonzepte des semestrierten Lehrplanentwurfs.

\section{4 Über Schwellenkonzepte zu den Basiskonzepten}

Eine definitorische Abgrenzung zwischen Basiskonzepten und Schwellenkonzepten scheint notwendig und Davies \& Mangan (2007) liefern einen vielversprechen-

Bei der Erschließung von Schwellenkonzepten im Unterricht ist immer das Primat der Kritikfähigkeit, der Multiperspektivität und der Kontingenz geboten ist. Im Falle des Eingangs erläuterten Beispiels iPhone, könnte das Schwellenkonzept der Wertschöpfungskette beispielsweise als gewichtiges Argument für und auch gegen das Phänomen des global sourcing eingesetzt werden. Dadurch wird einerseits die Dialektik der interessenspezifischen Verwendung von scheinbar neutralen (Schwellen)Konzepten deutlich und andererseits auch die performative Autorität, die sie in Bezug auf die Konstruktion von gesellschaftlichen Wirklichkeiten haben (vgl. Meyer \& Land 2003: 13). 
den Anhaltspunkt. Sie beschreiben Basiskonzepte ( $b a-$ sic concepts) als „, $[\mathrm{u}]$ nderstanding of everyday experience transformed through integration of personal experience with ideas from a discipline" (ebd.: 715). Wie Walker (2013: 248) es ausdrückt, werden Lernende mitsamt ihrem Alltagswissen abgeholt und in fachspezifische Grundbegriffe und Denkweisen eingeführt. Diese Definition ist durchaus mit der Anforderung an Basiskonzepte für GW aus dem Lehrplan (BGBl. 217/2016: $60 \mathrm{f}$.) kompatibel, die betont, dass Basiskonzepte eine erfahrbare und komplexe Welt durch fundamentale fachliche Ideen und Konzepte besser lesbar und verhandelbar machen sollen (z. B. Arbeit, Markt, Konsum).

Schwellenkonzepte hingegen bewegen sich ausschließlich innerhalb fachlicher Denkwelten. Sie werden von Davies \& Mangan (2007: 715) als höherstufiges ,understanding of other subject discipline ideas integrated and transformed through acquisition of theoretical perspective" bezeichnet oder, auf noch komplexerer Ebene als "[a]bility to construct discipline-specific narratives and arguments transformed through aquisition of ways of practising" (ebd.). Kennzeichnend für Schwellenkonzepte ist also die disziplininterne Erschließung von Denkweisen und Theorien, die das durch die Basiskonzepte des Lehrplans geforderte fachspezifische Verstehen der Welt überhaupt erst ermöglichen.

It is only once a transforming threshold concept has been subsequently incorporated into a learner's thinking that the full significance of a basic concept can really be grasped; they will have to revisit these concepts as they acquire the integrating framework to achieve a more sophisticated understanding. (Davies \& Mangan 2013: 4)

Es soll angemerkt werden, dass die Abgrenzung zwischen Schwellenkonzepten und Basiskonzepten im Falle des österreichischen Lehrplanentwurfs nicht immer feinsäuberlich möglich ist. So kann das Basiskonzept Kontingenz als Schwellenkonzept im Sinne der hier vorgeschlagenen Definition bezeichnet werden. Erst wenn es verinnerlicht wird, können die Welt und die Versuche sie erklärbar zu machen als potentiell veränderbar begriffen werden. Dem Prinzip der Kontingenz kommt damit die wichtige Funktion des Korrektivs gegenüber den anderen Basiskonzepten zu. Diese zielen jeweils auf den aktuellen Stand der Fachwissenschaft und es ist aus wissenschaftstheoretischer Sicht notwendig, ihre potentielle Veränderbarkeit zu verankern (vgl. Kuhn 1978). Da (in der Demokratie) die Durchsetzung von Veränderungen im Rahmen gesellschaftlicher Aushandlungsprozesse passiert, rückt durch das Basiskonzept Interessen, Konflikte und Macht das Schwellenkonzept der Diskursivität in den Vordergrund. Es geht darum, den Zusammenhang von Wissen und Macht zu erkennen und dabei Praktiken der Bedeutungszuschreibung als zentralen so- zialen Konfliktmechanismus zu begreifen (vgl. Said 1978; Glasze \& Mattissek 2009: 11).

Um Lernenden eine umfangreiche Vernetzung ökonomischer Zusammenhänge und kritischer Sichtweisen zu ermöglichen, sollten Basiskonzepte über faktische und ethische Schwellen des Verstehens erarbeitet werden (vgl. Mehren et al. 2015). Wachstum und Krise fordert die Bereitschaft neoklassische Erklärungspfade - inklusive der ihnen zu Grunde liegenden Schwellenkonzepte $-\mathrm{zu}$ verlassen und verlangt eine weiterführende kritische Einschätzung polit-ideologischer Konfliktlinien (vgl. Hinsch et al. 2014: 54). Ein ökonomistisches Verständnis von wirtschaftlichem Wachstum bedingt die Kenntnis grundlegender Indikatoren und Begriffe (endogenes Wachstum, exogenes Wachstum, BIP pro Kopf, Produktionsfaktoren, Investition, etc.) sowie komplexere Zugänge wie unter anderem Produktivität, Skalenerträge, Grenzkosten, Opportunitätskosten und Multiplikator-Effekte. Aus kritisch-sozialwissenschaftlicher Perspektive werden jedoch auch ganz andere Blickwinkel interessieren. Wenn wirtschaftliche Systeme Krisen hervorbringen, schaffen sie Gewinner/ innen und Verlierer/innen. Empowerment wäre ein denkbares Schwellenkonzept, das Schülerinnen und Schülern erlaubt, die Veränderung eines Ist-Zustandes nicht nur aus ökonomischer, sondern auch aus ethischsolidarischer Perspektive zu begreifen.

Natürlich geht es bei der Eröffnung von Schwellenkonzepten nicht darum, eine abstrakte Taxonomie der Konzepttypen zu entwerfen und diese dann anhand von Baumdiagrammen mechanisch abzuarbeiten. Vielmehr soll geklärt werden, welche grundlegenden disziplinären Schwellenkonzepte für die Beförderung bestimmter Basiskonzepte und GWspezifischer Denkweisen sinnvoll sein können; sowie welche Auswahl an Inhalten und Themen für diesen Zweck geeignet erscheint und dabei zum Erreichen der im Lehrplan geforderten kompetenzorientierten Lernziele beitragen kann. Aus der hier beschriebenen Sicht sind Schwellenkonzepte notwendige kognitive Bausteine für fachorientiertes, kritisch-differenziertes Denken. Nur wenn sie im Lernprozess anhand der passenden exemplarischen Inhaltsfindung erfolgreich entwickelt und eingesetzt werden, können Probleme auch selbstständig gelöst werden. Erst dann kann schließlich vom individuellen Erwerb spezifischer Kompetenzen (vgl. BMBF 2012) gesprochen werden.

\section{Schwellenkonzepte im Unterricht: Über- legungen und Anregungen zur Praxis GW}

Im Anschluss wird beispielhaft skizziert, wie Lehren und Lernen vor dem Hintergrund der Schwellenkonzepttheorie geplant werden kann, bzw. welche Kon- 
sequenzen das auf Lehr- und Lernarrangements im GW-Unterricht hätte. Zunächst wird erläutert, welche Arten von Wissen und Verstehen im Unterricht für die Beförderung von Schwellenkonzepten erstrebenswert sind. Perkins (2008: 13) betont diesbezüglich die Wichtigkeit von proaktiven Wissensformen (proactive knowledge) und zeigt, wie diese mit Schwellenkonzepten in Verbindung stehen. Proaktives Wissen beruht dabei in seiner Darstellung auf drei wesentlichen individuellen Faktoren. Erstens, tiefgreifendes Verständnis und Fähigkeit zur Anwendung des Gelernten; Zweitens, positive Einschätzung des Gelernten und Zuversicht in dessen Relevanz; Drittens, die Aufmerksamkeit, Offenheit und Bereitschaft, das Gelernte in unvorhersagbaren Problemsituationen einzusetzen. Schwellenkonzepte wirken sich positiv auf alle drei Faktoren aus (vgl. ebd.). Als kognitive Bausteine sind sie Grundlage von tiefgreifendem fachlichen Verständnis und begünstigen daher die Anwendung von Gelerntem (vgl. Davies \& Mangan 2013: 2). Schwellenkonzepte sind mitunter beschwerlich zu verarbeiten (vgl. Meyer \& Land 2003: 6) und verlangen stärkeres Kommittent im Lernprozess. Dadurch erhöht sich sowohl die Fähigkeit als auch die Bereitschaft sie autonom und situationsadäquat in Problemlösungsprozessen einzusetzen (vgl. Perkins 2008: 13).

In der Praxis wirkt sich die Ausrichtung auf Schwellenkonzepte wesentlich auf die Methoden und Inhalte des Unterrichts aus. Einerseits ist es wichtig, handlungsorientiertes und selbsttätiges, forschendes Lernen zu ermöglichen, auf der anderen Seite sollte kumulatives Lernen durch Inhalte gefördert werden, die die Erarbeitung von Schwellenkonzepten begünstigen (vgl. Baradell 2013; Meyer \& Land 2003; Davies \& Mangan 2013). Unterricht sollte dementsprechend als Angebot konzipiert werden (vgl. Helmke 2007), das eine möglichst vielfältige und variable Lernumgebung bereitstellt, anhand derer lebensweltorientiert Problemstellungen aufgegriffen und bearbeitet werden können. Schwellenkonzepte helfen Lernenden dabei, grundlegende fachliche Denkweisen zu verinnerlichen und anhand dieser Denkweisen gegenwärtige Probleme aus fachlich fundierter Perspektive zu beurteilen und zu bearbeiten. Daraus abgeleitete Erkenntnis hilft, weiterführende Schemen, Modellen und Theorien zu verbinden und zu vertiefen (vgl. Davies \& Mangan 2013: 4). Aus methodischer Sicht scheint es sinnvoll, die folgenden drei Aspekte (adaptiert nach Barradell 2013: 268) und vier Prinzipien (adaptiert nach Davies \& Mangan 2013: $7 \mathrm{f}$.) zu beachten, um die Bewältigung von Schwellenkonzepten im Unterricht zu erleichtern.

1. Lerninhalte sollen auf ihren transformativen Charakter sowie auf die, Beschwerlichkeit' im Zuge ihrer Erarbeitung überprüft werden. Wenn Lernende ein fachliches Konzept als schwierig einstufen, aber nach dem Begreifen des Konzepts eine Problemstellung besser lösen können, dann weist das auf die erfolgreiche Aneignung eines Schwellenkonzepts hin. Diagnostische Einblicke bieten sich hier durch die laufende Unterrichtsbeobachtung sowie durch Lehrer/innen/Schüler/innengespräche.

2. Lernschritte, die kognitive Schwellen überwinden, werden Lernenden dabei helfen fachlich vernetzter zu denken. Inhalte werden nach dem exemplarischen Prinzip (vgl. Wagenschein 1986) ausgewählt. Sie helfen Schwellenkonzepte initial zu erschließen und gewährleisten im Anschluss, dass fachliches Denken in steigender Komplexität befördert wird. Es geht vor allem darum ein verinnerlichtes Schwellenkonzept, wie beispielsweise die Wertschöpfungskette, anhand verschiedener Szenarien zu testen und dabei selbst lebensnahe Problemstellungen zu identifizieren, wo das Konzept sinnvoll zu weiteren Erkenntnissen führen kann.

3. Der transformative und irreversible Charakter von Schwellenkonzepten führt auch dazu, dass Lernende sich und die Welt anders sehen und verstehen. Das setzt mitunter schmerzhafte und emotional fordernde Lernerfahrungen voraus, bei denen Lernende begleitet werden sollten. Andernfalls ist zu befürchten, dass Lernende Angst oder Ablehnung empfinden und sich gegen bestimmte fachliche Sichtweisen wehren.

Die folgenden vier Prinzipien erscheinen darüber hinaus für die unterrichtspraktische Umsetzung relevant:

1. Variation in der Bereitstellung an Lernmöglichkeiten in Hinblick auf Methoden, Materialien und Lernformen. Da Schwellenkonzepte elementare Erkenntnismomente (vgl. Klafki 1963) auf kognitiver Ebene (vgl. Walker 2013) befördern, sollte eine vielfältige und lebensweltorientierte Lernumgebung sinnvolle Transferleistungen unterstützen.

2. Nähe zu wissenschaftlichen Methoden und ihrer Umsetzung in der Lebenswelt. Lernen soll als individueller Prozess des Forschens verstanden werden, der zu Modell- und Konzeptbildung führen kann. Lernende erarbeiten entsprechend Lösungswege über disziplinäre Denkweisen, die zur Erklärung von Phänomenen in der realen Welt verwendet werden können.

3. Reflektion von Alltagswissen und Abgleich mit fachlichen Erklärungsansätzen. Dabei werden wissenschaftliche Sichtweisen mit den Vorstellungen der Lernenden abgeglichen, um lernförderliche Erschließungswege zu eröffnen (vgl. Kattmann et al. 1997). Vorhergehendes Wissen soll aber nicht als falsch gelten, sondern als notwendig und adaptierbar verstanden werden.

4. Die Einsicht, dass auch fachliche Erkenntnis kon- 
tingent ist und damit die Bereitschaft zu erlangen, dass lebenslanges Leben notwendig ist: Lebenslanges Lernen kann dementsprechend selbst als wichtiges Schwellenkonzept verstanden werden.

Die folgende Lernaktivität ist für eine 8. Klasse AHS konzipiert und greift fachlich schwerpunktmäßig auf die beiden Basiskonzepte Märkte, Regulierung und Deregulierung sowie auf Interessen, Konflikte und Macht zu. Das vorgeschlagene Szenario weist Elemente des forschenden Lernens (vgl. Wildt 2009) auf und setzt problemorientierte und offene Lernformen voraus. Anregungen kommen aus dem Handbook for Economic Lecturers (Davies \& Mangan 2013) und wurden von mir entsprechend für den österreichischen GW-Unterricht adaptiert. Auf authentische Lernszenarien wird bei der Umsetzung geachtet (vgl. Meyer \& Land 2005: 384). Des Weiteren findet das lerndiagnostische Instrument der Metakognition Anwendung (vgl. Bransford et al. 2000). Die Schüler/innen arbeiten entweder in Einzelarbeit oder in Kleingruppen und verfügen über entsprechende Vorkenntnisse. Adäquate Recherchematerialien stehen bei Bedarf zur Verfügung. Die Lernaktivität geht von einer aktuellen Problemstellung aus dem Interessensbereich von GW aus und beschreibt vier Phasen der Erarbeitung.

1. Im ersten Schritt geht es um die Reflektion des fachlichen Vorwissens, um dessen zielgerichteten Ausbau durch Recherche sowie den Transfer hin zur Problemstellung. Die Lernenden sollen eine fachlich begründete Antwort auf folgende Forschungsfrage geben:

\section{Welche Auswirkungen kann protektionistische US-Außenhandelspolitik auf die USA und ihre wichtigsten Handelspartner haben?}

Aufgabe ist es, die Situation in eigenen Worten zu erklären und die verschiedenen Aspekte (ansatzweise) aus ökonomischer Perspektive zu beleuchten. Differenzierte Erörterungen der Fragestellungen deuten auf eine erfolgreiche Verinnerlichung entsprechender fachspezifischer Denkweisen hin.

2. Im zweiten Schritt folgt die Abstraktion und Verankerung des Gelernten im fachlichen Diskurs. Schüler/innen bekommen eine Liste mit ihnen voraussichtlich bereits bekannten ökonomischen Schwellenkonzepten ${ }^{2}$, die für die Beantwortung

\footnotetext{
2 Die hier aufgelisteten Vorschläge nehmen Anlehnung an von Davies \& Mangan (2013: 4) und Fouberg (2013: 68) vorgeschlagenen ökonomischen und geographischen Schwellenkonzepten und sind vom Autor anhand der fünf Kriterien von (Meyer $\&$ Land 2003) in Hinblick auf die Fragestellung und die mehrperspektivischen Anforderungen der Basiskonzepte des Lehrplans adaptiert worden.
}

der Forschungsfrage in Punkt 1 relevant sein können. Es muss dazu angemerkt werden, dass es keine endgültige und ,richtige' Auswahl von Schwellenkonzepten gibt, die für alle Lernenden (z. B. einer Klasse) zutreffend ist und die sie gemeinsam auf einem linearen Lernweg durch ein Basiskonzept führt. Walker (2013: 249) weist darauf hin, dass individuelle kognitive Schwellen disziplinären Denkens auf ganz unterschiedlichen Komplexitätsniveaus angesiedelt sein können und daher nicht problemlos allgemein identifiziert werden können. Der lerndiagnostische Ansatz der Metakognition erscheint in der Praxis vielversprechend um Schwellenkonzepte transparenter zu machen und Schüler/innen und Lehrer/innen ein Bild darüber zu verschaffen, wann und wie welche Hürden fachlichen Denkens tatsächlich überwunden werden (vgl. Fouberg 2013). Lernende reflektieren dabei ihren individuellen Lernprozess durch geeignete Arbeitsaufträge. Sie machen sich bereits verinnerlichte Schemen bewusst, bauen diese bei Bedarf aus oder revidieren sie (Bransford et al. 2000: $18 \mathrm{ff}$. .). Lehrende sind entsprechend gefordert, Basiskonzepte in ihrer Prozesshaftigkeit zu begreifen und diese über grundlegende Konzeptschritte ökonomischen Denkens zu eröffnen. Die nachfolgenden Aktivitäten greifen teilweise auf Techniken der Metakognition zurück.

Um zu testen, ob die Schüler/innen die aufgelisteten Schwellenkonzepte bereits verinnerlicht haben, beziehungsweise um zu erkennen, ob es ihnen möglich ist, diese problemorientiert verfügbar zu machen,werden sie um eine beispielhafte Erklärung der Schwellenkonzepte gebeten. Anschließend werden Kärtchen ausgeteilt, mit deren Hilfe die eigene Erläuterung durch eine Zusammenfassung der Kernidee/ eine Karikatur bestätigt oder erweitert wird.

- komparative Kostenvorteile

- Effizienz

- Spezialisierung im Außenhandel

- Opportunitätskosten

- Wertschöpfungskette

- Grenzkosten / Grenzerlös

- Skaleneffekte

- Standortfaktoren

- Substitutionsgüter

- Angebot und Nachfrage

- Konsumpräferenzen

- Subvention

- Supermacht

- Zentrum \& Peripherie

- Disparität

- Diskursivität 


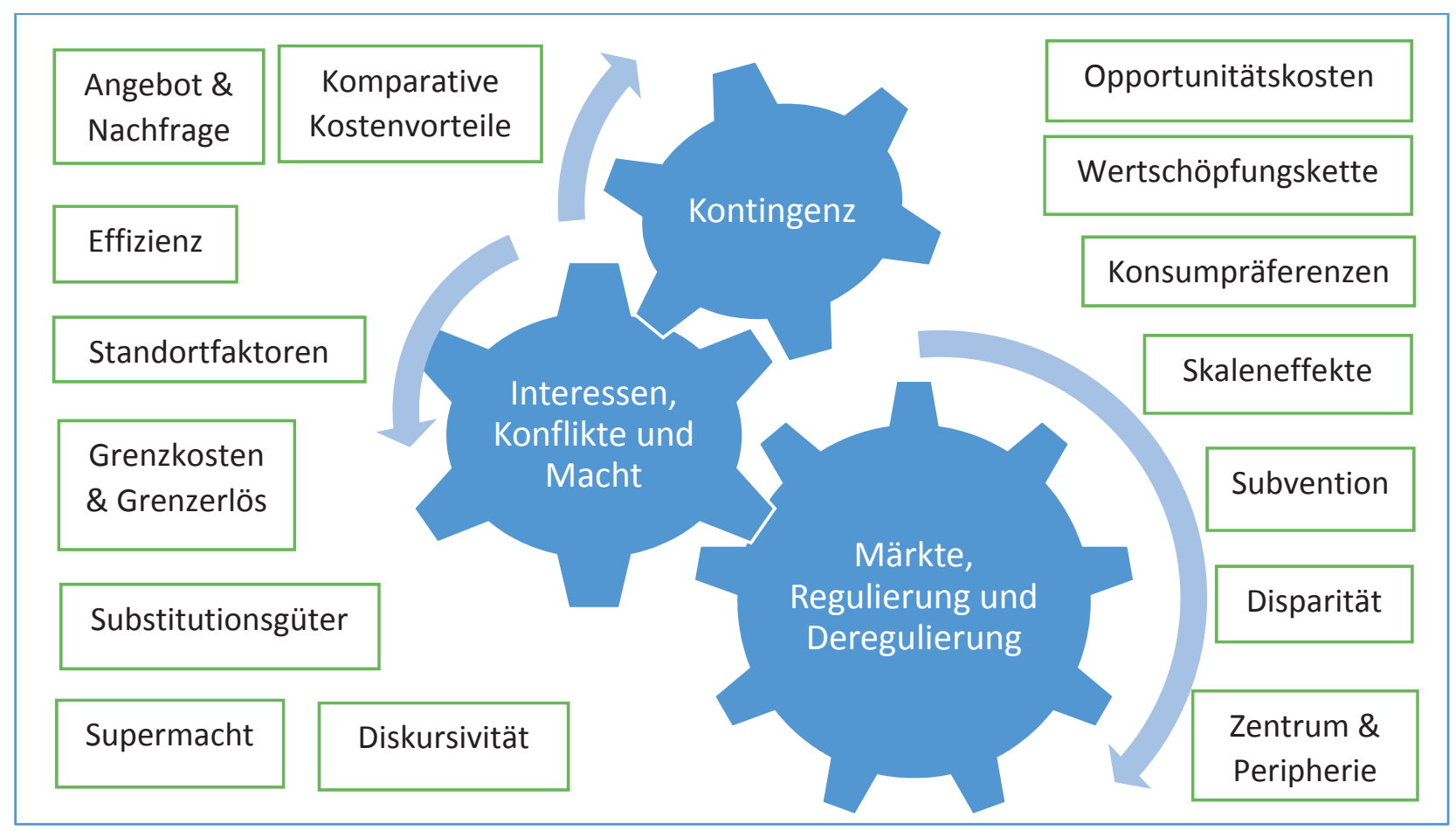

Abb. 1: Schwellenkonzepte und ausgewählte Basiskonzepte

Die Schüler/innen sollen nun eines oder mehrere dieser Schwellenkonzepte auswählen und in einem kurzen Text erklären, wie und warum es ihnen dabei hilft, die Problemstellung aus GWspezifischer Sicht zu erfassen und kritisch zu beurteilen. Hier wird vor allem deutlich, welche Schwellenkonzepte zur Anwendung kommen, in welchem Umfang sie verstanden wurden, und wie weit Lernende sie miteinander vernetzen können.

3. Im dritten Schritt geht es um den Transfer und proaktiven Einsatz des Gelernten. Die Schüler/ innen sollen aus der Liste der Schwellenkonzepte (siehe oben) ein bis drei auswählen und dazu eine Fragestellung formulieren, die ein anderes, ebenfalls selbst gewähltes (aktuelles) ökonomisches Phänomen aus dem Bezugsbereich der beiden Basiskonzepte Märkte, Regulierung und Deregulierung sowie Interessen, Konflikte und Macht aufgreift. Sie sollen dabei begründen, warum sie bestimmte Schwellenkonzepte gewählt haben und ansatzweise beschreiben, welche Erklärungsperspektiven ihnen diese eröffnen. Auf diese Weise lässt sich erkennen, ob Schüler/innen auf proaktive und kreative Weise mit ihrem Wissen umgehen können und verinnerlichte Schwellenkonzepte auch problemorientiert verfügbar machen können.

4. Abschließend werden die thematisierten Schwellenkonzepte individuell und im Plenum besprochen. Dieser Schritt scheint zur Sicherung der Lernerträge notwendig und lässt es zu, die Über- schneidungen und individuellen Wege der Aneignung der einzelnen Schwellenkonzepte auf dem Weg zu umfangreicheren fachlichen Denkens zu reflektieren. Lernende und Lehrende können die von Meyer \& Land (2003) angeführten Kriterien (transformativ, irreversibel, integrativ, grenzorientierend und beschwerlich) mit jenen Schwellenkonzepten abgleichen, die sie gerade eingesetzt haben. Das kann durch eine Mindmap geschehen, die ihren persönlichen Weg über ein bestimmtes Schwellenkonzept hin zur disziplinspezifischen Betrachtung eines Phänomens darstellt.

Wie die Lernaktivität zeigt, verlangt die Beförderung von Schwellenkonzepten im Unterricht eine zumindest bedingte Auflösung traditioneller Lehr- und Lernformen. Dazu bedarf es vor allem hoher fachlicher Kompetenz der Lehrkraft, wenn diese spontan auf von den Lernenden entwickelte Fragestellungen eingehen soll. Der Unterricht orientiert sich an einem realweltlichen Problem, das aus fachlicher Perspektive differenziert erschlossen wird. Vorwissen wird selbstständig verfügbar gemacht, individuell erweitert und situationsadäquat eingesetzt. Konzepte werden anhand ihrer unmittelbaren Einsetzbarkeit erarbeitet und auf andere Ereigniskontexte übertragen. Das Überwinden kognitiver Hürden wird demgemäß zu einer Anstrengung, die Lernende mit einer tiefgreifenden Problemlösungskompetenz belohnt. Schüler/innen lernen fachlich zu denken, indem sie fachlich denken und nicht indem sie lernen, wie sie fachlich denken sollen. 


\section{Conclusio und Ausblick}

Weiterführende Forschung zur Schwellenkonzepttheorie scheint im Bereich der GW-Fachdidaktik vor allem in zwei Bereichen vielversprechend. Erstens wäre es sinnvoll, empirisch zu klären, welche ökonomischen und geographischen Schwellenkonzepte den Schülerinnen und Schülern mehrheitlich dabei helfen, bestimmte Basiskonzepte umfangreicher zu begreifen und zweitens wäre es zielführend eine grundlegende didaktische Anknüpfung der Schwellenkonzepte an die Basiskonzepte des semestrierten Lehrplans zu entwerfen und dabei auch umfangreich an den internationalen Forschungsstand zu ökonomischen und geographischen Basiskonzepten anzuschließen. Aus Platzgründen wurde in dieser Publikation darauf verzichtet und der Schwerpunkt auf die Schwellenkonzepttheorie gelegt. Das Ziel dieses Artikels ist es, einen ersten Impuls zur Aufnahme des Schwellenkonzeptansatzes in den Diskurs der österreichischen GW-Didaktik zu liefern und mögliche Anregungen für die Unterrichtspraxis sowie für weiterführende Forschungsrichtungen aufzuzeigen.

\section{$7 \quad$ Literatur}

Barradell, S. (2013): The Identification of Threshold Concepts: A Review of Theoretical Complexities and Methological Challenges. Higher Education 65. S. 265-276.

BGBl. 219 (2016): Verordnung der Bundesministerin für Bildung, mit der die Verordnung über die Lehrpläne der allgemein bildenden höheren Schulen geändert wird. https:// www.ris.bka.gv.at/Dokumente/BgblAuth/BGBLA_2016_ II_219/BGBLA_2016_II_219.pdf (27.7.2017).

Bischoff, J. (2009): Jahrhundertkrise des Kapitalismus Abstieg in die Depression oder Übergang in eine andere Ökonomie. Hamburg: VSA.

BMBF (2012): Die kompetenzorientierte Reifeprüfung aus Geographie und Wirtschaftskunde - Richtlinien und Beispiele für Themenpool und Prüfungsaufgaben. https:/www.bmb.gv.at/schulen/unterricht/ba/reifepruefung_ahs_lfgw_22201.pdf?5i84h4 (23. 9. 2016).

Bransford, J., A. Brown \& R. Cocking (Hrsg., 2000): How people learn: Brain, mind, experience, and school. Washington: National Academy Press.

Carey, S. (1991): Knowledge acquisition: enrichment or conceptual change? In: Carey, S. \& R. Gelman. (Hrsg.): The epigenesis of mind: essays on biology and cognition. New York: Lawrence Erlbaum Associates. S. 459-487.

Cousin, G. (2008): Threshold Concepts: Old Wine in New Bottles or a New Form of Transactional Curriculum Inquiry. In: Meyer, J., R. Land \& J. Smith (Hrsg., 2008): Threshold Concepts within the Disciplines. Rotterdam: Sense Publishers. S. 261-272.

Davies, P. \& J. Mangan (2007): Threshold Concepts and the Integration of Understanding in Economics. Studies in Higher Education 32/6. S. 711-726.
Davies, P. \& J. Mangan (2013): The Handbook for Economics Lecturers. Economics Network. http://www.economicsnetwork.ac.uk/handbook/printable/threshold_concepts.pdf (27.7.2017).

Fouberg, E. (2013): „The world is no longer flat to me“: student perceptions of threshold concepts in world regional geography. Journal of Geography in Higher Education 37/1. S. 65-75.

Fridrich, C. \& M. Hofmann-Schneller (2017): Positionspapier "Sozioökonomische Bildung“. GW-Unterricht 145. S. 54-57.

Glasze, G. \& A. Mattissek (2009): Diskursforschung in Humangeographie: Konzeptionelle Grundlagen und empirische Operationalisierungen. In: ebd. (Hrsg.): Handbuch Diskurs und Raum. Bielefeld: Transcript. S. 11-60.

Helmke, A. (2007): Guter Unterricht - nur ein Angebot. Friedrich Jahresheft 2007. S. 62-65.

Hedtke, R. (2015): Sozioökonomische Bildung als Innovation durch Tradition. GW-Unterricht 140. S. 18-38.

Hinsch, S., H. Pichler, T. Jekel, L. Keller \& F. Baier (2014): Semestrierter Lehrplan AHS, Sekundarstufe II. Ergebnis der ministeriellen Arbeitsgruppe. GW-Unterricht 135. S. 51-61.

Jonen, A., K. Möller, \& I. Hardy (2003): Lernen als Veränderung von Konzepten - am Beispiel einer Untersuchung zum naturwissenschaftlichen Lernen in der Grundschule. In: Cech, D. \& H.-J. Schwier (Hrsg.): Lernwege und Aneignungsformen im Sachunterricht. Bad Heilbrunn: Klinkhardt. S. 93-108.

Kattmann, U., R. Duit, H. Gropengießer \& M. Komorek (1997): Das Modell der Didaktischen Rekonstruktion. Zeitschrift für Didaktik der Naturwissenschaften 3. S. 3-18.

Klafki, W. (1963): Das pädagogische Problem des Elementaren und die Theorie der kategorialen Bildung. Weinheim: Beltz.

Klafki, W. (1994): Zweite Studie: Grundzüge eines neuen Allgemeinbildungskonzeptes. Im Zentrum: Epochaltypische Schlüsselprobleme. In: ebd.: Neue Studien zur Bildungstheorie und Didaktik. Zeitgemäße Allgemeinbildung und kritisch-konstruktive Didaktik. Weinheim/ Basel: Beltz. S. 43-82.

Kuhn, T. (1970): The Structure of Scientific Revolutions. Chicago: University of Chicago Press.

Mehren, M., R. Mehren, U. Ohl, \& C. Resenberger (2015): Die doppelte Komplexität geographischer Themen: Eine lohnenswerte Herausforderung für Schüler und Lehrer. Geographie aktuell \& Schule 37/216. S. 4-11.

Meyer, J. \& R. Land (2003): Threshold Concepts and Troublesome Knowledge 1: linkages to ways of thinking and practising within the disciplines. In: Rust, C. (Hrsg.): Improving Student Learning: Improving Student Learning Theory and Practice - Ten Years On. Oxford: Oxford Centre for Staff and Learning Development. S. 1-16.

Meyer, J. \& R. Land (2005): Threshold Concepts and Troublesome Knowledge (2): Epistemological Considerations 
and a Conceptual Framework for Teaching and Learning. Higher Education 49/3. S. 373-388.

Perkins, D. (2008): Beyond Understanding. In: Meyer, J., R. Land \& J. Smith (Hrsg., 2008): Threshold Concepts within the Disciplines. Rotterdam: Sense Publishers. S. 3-20.

Picketty, T. (2015): About Capital in the Twenty-First Century. American Economic Review: Papers \& Proceedings 105/5: S. 48-53.

Rhode-Jüchtern, T. (2011): Die andere Intelligenz: Kausalität oder Kontingenz? http://www.geographie.uni-jena. de/geogrmedia/Lehrstuehle/Didaktik/Personal/Rhode_Juechtern/Vortr\%C3\%A4ge/Daten+ab13_12_11/ Die+andere+Intelligenz+Text.pdf (27.7.2017).

Said, E. (1978): Orientalism. London: Routledge.

Vielhaber, C. (1999): Vermittlung und Interesse. Zwei Schlüsselkategorien fachdidaktischer Grundlagen im Geographieunterricht. In: Vielhaber, Christian (Hg.):
Geographiedidaktik kreuz und quer. Vom Vermittlungsinteresse bis zum Methodenstreit, von der Spurensuche bis zum Raumverzicht. Wien. S. 9-26 (= Materialien zur Didaktik der Geographie und Wirtschaftskunde 15)

Vielhaber, C. (1988): Perspektiven einer kritisch-emanzipatorischen Fachdidaktik Geographie. Habilitationsschrift an der Grund- und Integrativwissenschaftlichen Fakultät der Universität Wien, Wien.

Weber, B. (2015): Editorial: Multiperspectivity, Values and Criticism in Economic and Civic Education. Journal of Social Science Education 14/4. S. 2-6.

Wagenschein, M. (1968): Verstehen lehren. Genetisch Sokratisch - Exemplarisch. Weinheim: Beltz.

Walker, Guy. 2013. A Cognitive Approach to Threshold Concepts. Higher Education 65/2. S. 247-263.

Wildt, J. (2009): Forschendes Lernen: Lernen im „Format“ der Forschung. Journal Hochschuldidaktik. Forschendes Lernen: Perspektiven eines Konzepts 20/2. S. 4-7. 